\title{
GRASP Combined with ILS for the Vehicle Routing Problem with Time Windows, Precedence, Synchronization and Lunch Break Constraints
}

\author{
Ettazi Haitam ${ }^{1}$, Rafalia Najat ${ }^{2}$, Jaafar Abouchabaka ${ }^{3}$ \\ LaRI Laboratory, Faculty of Sciences, University Ibn Tofail ${ }^{1,2,3}$ \\ Kenitra, Morocco
}

\begin{abstract}
In this era of pandemic especially with COVID-19, many hospitals and care structures are at full capacity regarding availability of beds. This problem leads to ensure giving specific cares to people in need either in illness or disability in their own homes. Home Health Care (HHC) proposes this kind of services for patients demanding it. These services have to be done at the request of the patient which appears to be the client in a way that gives satisfaction to the requester of the service. Often, these demands are bound by a specific time that the workforce (caregivers) are obligated to respect in addition to the precedence (priority) constraint. The main purpose of the HHC structures is to provide a service that is good in term of quality, minimize the overall costs and shorten the losses. To reduce the costs of these HHC structures, it is mandatory to find comprehensible and logical ways to do it, for it is not permissible to touch the caregiver salary, HHC structures find themselves in the obligation to optimize by other means such as reducing the travel cost. Note that these structures give cares in one's home, which means that the travel aspect is important and is considered the core spending charges of the institution. Another fact is the satisfaction of the patients toward caregivers; this is an essential element to optimize in order to obtain a good quality service, to give a realistic aspect for the problem the lunch break of caregivers is introduced as a parameter. For those arguments, a conception of an efficient planning of caregivers involves using decision tools and optimization methods. A caregiver (vehicle) is attributed to a patient (customer) to do a number of cares with several options in accordance to the customer wishes like time windows requirement often specified by the client, the priority or precedence constraints are usually performed if a care have to be performed before another and could need the intervention of more than one caregiver and must have at least one lunch break a day and it is not always taken at a set time of the day and must be versatile to optimize customer demand satisfaction. To resolve this issue which is called VRPTW-SPLB, a mathematical model of the problem is proposed and explained as a Mixed Integer Linear Programming (MILP) and a greedy heuristic based on a Greedy Randomized Adaptive Procedure (GRASP) is proposed, two strategies based on local search and two metaheuristics, and a metaheuristic resultant of an hybridization of the two metaheuristics. At the end of the paper, results are shown on a benchmark extracted from the literature.
\end{abstract}

Keywords-Optimization; VRP; home health care; ILS; tabu search; metaheuristics

\section{INTRODUCTION}

The world nowadays know a considerable increase in growing rate of population along with the several diseases that emerges day by day leading to a decrease in the number of beds in hospital per person. This situation arises many problems such offering adequate cares to needy people without delay. The Home Health Care services (HHC) becomes more complex and must adapt and evolve to cover all this variables by applying decisions in operational research context. To identify the source of the problem and its solution, defining the major actors is essential, these services are made essentially for disabled/elderly people that request many needs like medical care, hygienic care, assistance... In order to fulfill these demands, HHC structures offer the opportunity to perform such care in patient's homes along with the adequate and necessary equipment and resources may be caregivers or devices and gives the patient's the feeling that they are treated like if they receive treatment in a traditional hospital. This overall situation obligate to create a fine and good collaboration and coordination between human and material resources in order to establish a good and optimized planning that increases the quality of services offered by home health care with a controlled and reduced generated costs.

In this study, a generalization of the classical problem known as vehicle routing problem is proposed to deal with special and particular aspects linked to HHC. The problem studied in this paper consists of generating a set of routes executed by a group of vehicles (qualified caregivers) in order to visit a number of dispatched clients (patients) while satisfying a set of preferences aspired by the patients towards caregivers so that the operation is done in the most effective way. To enrich this study, the cares (2 or more) are of two categories according to the synchronization aspect, either simultaneously (at the same time) or in a given order(precedence), this case need the coordination of a high level of caregivers. To give the problem a realistic aspect that most of the studies neglect in their work is by considering the lunch break of caregivers which are operated only after the termination of a care by a caregiver.

To summarize what is being said, the problem studied is called the Vehicle Routing Problem with Time Windows, Synchronization, Precedence and Lunch Break constraints (VRPTW-SPLB). The constraints applied in this generalization of the VRP is appropriate to the maximum as it 
is in real life where most of patients have a preferred time to not precede or exceed, two or more cares can be done by more than one caregiver according to the skills of this latter, the cares can be one after another according to the priority of care and finally caregivers need the replenish their energy to continue the cares in the most efficient way thus the need to have a lunch break granted to the caregiver.

The next sections of this article are structured as follows: a section is devoted to the literature review of past works dealing with the vehicle routing problem in the home health care sector, the next section is dedicated to the problem definition, afterward a mathematical formulation is proposed in Section 4, next the explanation and components of the different metaheuristics used to solve the problem. Section 6 illustrates the computational results, while Section 7 is mainly for the discussion. Finally a conclusion and future perspectives are displayed.

\section{LITERATURE REVIEW}

The vehicle routing problem is regarded as an NP-Hard problem and considered within the field of Operational Research as one of the most studied in the last two decade. In order to assimilate a valid and explicit explanation of the vehicle routing problem as proposed by [1] is to build a route delivery with the least possible cost from a center (depot) to a series of distributed locations (customers) in accordance to a set of constraints that need to be respected. Both exact and approximate methods or approaches are considered as solution to the problem, only that metaheuristics methods are plainly approved to be effective as illustrated in [2] which is up to find a solution up to 26 variants of the VRP. The main body of this study is to plan and allocate several resources such as vehicles (caregivers), material... to a set of demanding requests made by a group of clients (patients) dispatched in a geographical region to achieve a predefined and specific services. From that perspective, the arousal of many constraints seem natural such as fixed time windows determined by the patient, preferences of patients toward caregivers, synchronization of services either simultaneously or not, lunch break of caregivers... Many approaches set by many authors such as [3-7] have realized and confirmed that the best methods to solve this problem is by using heuristics and metaheuristics. These authors experimented their approaches on qualified and known benchmark of the literature offered by $[8,9]$. Putting all this aside, it is obligatory to search and evaluate the most common objectives (cost, preferences, waiting time...) considered in those studies. Considering the HHC context, the vehicle routing problem offers as a problem many objectives to optimize; the total travelled distance by vehicles, the number of vehicles required, the total travelled time, patient's preferences toward caregivers are the most studied as objectives to optimize and frequently studied separately, thus these objectives can be considered on together but very rarely.

The idea supported in transportation in the home care as a logistical issue can be clustered into two broad categories, allocation and planning issues and pickup and delivery issues.

In this paper, the rest of the literature proposed in this study will be separated in three subsections, studies and works illustrated in the past talking on the subject of vehicle routing problem in home health care sector are shown in first, the second is an extension of contribution linked to the aspect synchronization and precedence in the vehicle routing problem and the last section is a highlights of the current contribution.

\section{A. VRP in HHC}

The logistics factor was first demonstrated in the field of Health Services by [10-12] on door-to-door transportation to support the elderly and/or disabled. The increasing rate of elderly people worldwide has prompted the authorities to introduce a scheme called DARP that translates to Dial-ARide in order to assist people with disabilities of any type. From this perspective, [13,14] have established a particular version of DARP that seeks to deploy a special team with appropriate and specific expertise to the home of the patient to assist them with any support they request (bath, dress, help to move...) until the resource that is responsible for transporting them to the hospital or a treatment structure arrives. This scenario has prompted researchers to integrate constraints such as synchronization constraints to ensure that priority between visits is respected, in order to ensure the effectiveness of this procedure, fine teamwork is expected by medical assistants and transporters. In the same category, a new constraint emerges for patient requirements that allow them to select a time window in which they are prepared to receive the care staff in their homes. The author in [15] suggests and incorporates this feature of time windows in the DARP for improved planning, but does not take into account the state of emergency and is instead concerned with minimizing the cost of transporting patients. The Dial-A-Ride problem has been expanded into the HHCP (Home Health Care Problem) conducted by a study well known in the literature [16] in which the key concept is to delegate caregiver staff to provide a certain amount of care needed by clients (patients) in their homes. The study shows two methods of resolution, the first of which was an exact method by suggesting a mathematical model with inter mixed variables (MILP) and the other one is a heuristic method. As for applying these methods on real instances, 10 patients and 4 nurses were considered to do the test, this will be the initial step to various and many papers addressing the same study but by using and testing various optimization resources and tools.

The optimization context in the home health care sector considers many aspects. The logistical aspect as concerned with this study contains two main categories that can be illuminated regarding other categories such as planning and allocation issues and pickup and delivery issues which are considered to be the most illustrated aspect on this field. For this study to be comprehensive and for the sake of simplification of the problem, generally a patient asking for a required care service is allocated a caregiver according to adequate skill and qualification for doing and fulfilling the service. Those skills and qualification are medical qualification, age, gender, language, specialty... The caregiver has a begin time and an end time throughout the day. As for the service required, it is characterized by a duration, skill and time window. 
The dilemma of attributing visits to personal caregivers and the preparation of visits was studied in[17], where a MILP methodology and a Tabu search method were implemented to solve the problem and deliver fair and high quality solutions. In the same line, [18] proposed the same approach but considered as a partitioning problem which goal is to maximize the satisfaction of the patients and to minimize the transportation duration. There are studies in the literature that considers a single objective optimization such as [19] where the time windows constraint was introduced therefore the problem became VRPTW (Vehicle Routing Problem with Time Windows) and proposed a metaheuristics based on VNS and a mathematical formulation (MILP) in order to shorten the time that nurses consume in their movements and to maximize and increase the ratio nurses/patients of satisfaction. Another study [20] aims to solve the vehicle routing problem with time windows but this time with another approach and another implementation by introducing the Particle Swarm Optimization (PSO) so that the total travelled distance by nurses and caregivers is reduced with respect of constraints of time windows and vehicle's capacity. The multi-objective optimization is another variant of dealing with optimization in HHC, considering two functions to optimize at the same time leading to a high complexity of the problem.

The study in [21] deals with this multi-objective optimization by applying two metaheuristics based on ACO (Ant colony Optimization) which is an algorithm based on population, a mathematical formulation for the linear optimization and a multi-objective genetic approach.

Coordination of visits between nurses and/or caregivers is considered in the study of $[22,23]$. This coordination is either simultaneously or in a given order. The authors proposed a Branch and Bound method in order to reduce the travel cost of caregivers and to increase and maximize the satisfaction of patients toward nurses/caregivers. As for waiting time, which is a significant matter to consider was addressed by [24] for the sake of planning and scheduling visits to patients and giving tasks and mission to nurses/caregivers. First the mathematical formulation was established. Some authors tackled this problem by solving it in stages and not fully at once. This was done by $[25,26]$ where a set of routes were generated initially before assigning routes to caregivers then enforce synchronization constraints in order to reach feasible and concrete solutions. A problem of node duplication was addressed by [27] where to run away from this duplication of nodes, an Iterated Local Search (ILS) was implemented and applied on relatively small instances of the literature. As for [28], maximizing the satisfaction of patients was prioritized along with the reduction of the cost of travel by using a Variable Neighborhood Search (VNS).

Synchronization constraints are really important to highlight the VRPTW problem in its totality where a set of request made by patients can have multiple visits and require the intervention of one or several caregivers either simultaneously or in a predefined order. In [7], work on this by formulating at first a mathematical skull of the problem, a GRASP and several metaheuristics for the sake of optimizing the total travel cost and increase the satisfaction of patients.
The pick-up and delivery aspect is studied and highlighted as well in many papers where a patient is to be picked up from a place or dropped off in a place. This was highlighted by [13]. This aspect of picking up and delivering is flexible to the points that authors in [4] proposed a system in order to deliver meals to patient's homes. To do so, patients were clustered and partitioned in zones according to the care structures policy. Meanwhile caregivers were also grouped in teams.

Before jumping on the constraints used in general, detailing synchronization constraints on the home health care sector is significant to the continuity of this study. As for our knowledge, the synchronization aspect dealing with the home care services are either considered in a simultaneous way likewise [27]. Other authors considered both simultaneous and precedence (in a given order) in separate, this is clearly shown in [23] and [14] where [7] and [29] illustrated the synchronization in the same model. In our study, we will consider both synchronization aspect in addition to a set of constraints that were not combined in the same study before.

Table II shows the overall constraints of paper found in literature meanwhile Table I expresses the constraints that are the most common.

TABLE I. CONSTRAINTS COMMONLY USED IN HHC

\begin{tabular}{|ll|}
\hline Abbreviation & Description \\
\hline TW & Time Windows \\
Simu & Simultaneous synchronization \\
Prec & Precedence synchronization \\
SS & Specific Service \\
LB & Lunch Break \\
\hline
\end{tabular}

TABLE II. CONSTRAINTS IN THE LITERATURE

\begin{tabular}{|l|l|l|l|l|l|l|}
\hline Category & Article & TW & Simu & Prec & SS & LB \\
\hline & {$[17]$} & & & & $\mathrm{X}$ & \\
\hline & {$[18]$} & $\mathrm{X}$ & $\mathrm{X}$ & & $\mathrm{X}$ & \\
\hline & {$[20]$} & $\mathrm{X}$ & & & & \\
\hline & {$[23]$} & $\mathrm{X}$ & $\mathrm{X}$ & $\mathrm{X}$ & & \\
\hline $\begin{array}{l}\text { Planning \& } \\
\text { Scheduling }\end{array}$ & {$[29]$} & $\mathrm{X}$ & & $\mathrm{X}$ & $\mathrm{X}$ & \\
\hline & {$[25]$} & $\mathrm{X}$ & $\mathrm{X}$ & & & \\
\hline & {$[27]$} & $\mathrm{X}$ & $\mathrm{X}$ & & $\mathrm{X}$ & \\
\hline & {$[30]$} & $\mathrm{X}$ & $\mathrm{X}$ & $\mathrm{X}$ & & \\
\hline & {$[31]$} & $\mathrm{X}$ & $\mathrm{X}$ & $\mathrm{X}$ & $\mathrm{X}$ & \\
\hline & {$[16]$} & $\mathrm{X}$ & & & $\mathrm{X}$ & \\
\hline & {$[15]$} & $\mathrm{X}$ & & & & \\
\hline & {$[32]$} & $\mathrm{X}$ & $\mathrm{X}$ & $\mathrm{X}$ & & \\
\hline $\begin{array}{l}\text { Pick-up \& } \\
\text { Dekiveries }\end{array}$ & {$[33]$} & $\mathrm{X}$ & & & & \\
\hline & {$[34]$} & $\mathrm{X}$ & & & & $\mathrm{X}$ \\
\hline & {$[14]$} & $\mathrm{X}$ & & $\mathrm{X}$ & & \\
\hline $\begin{array}{l}\text { Both } \\
\text { categories }\end{array}$ & & & & & & \\
\hline
\end{tabular}




\begin{tabular}{|l|l|l|l|l|l|l|}
\hline & {$[35]$} & $\mathrm{X}$ & & & $\mathrm{X}$ & \\
\hline Our paper & & $\mathrm{X}$ & $\mathrm{X}$ & $\mathrm{X}$ & $\mathrm{X}$ & $\mathrm{X}$ \\
\hline
\end{tabular}

As shown in Table II, the temporal aspect is what most of the authors are interested in for the aim to get a better quality of services. In the other hand, the constraints that give a realistic aspect to the problem are neglected in the most of the works such as lunch break of caregivers, precedence constraints and synchronization constraints.

\section{B. VRP with Synchronization Constraints in HHC}

When talking about timing and temporal constraints in the vehicle routing problem especially in the home health care sector, it is very needy to point at very important notion that enforce the realistic aspect of the problem overall. the study in [36] point in his classification that there is a dependence that takes place in the route taken by caregivers in order to get to patient's homes requesting cares, he defines it as synchronization. This starting point encourage other authors such as [27] to group and cluster this so-called synchronization into two main part, one for the simultaneous synchronization which is a two or more than one care is done at the same time, this type of synchronization require the intervention of more than one caregivers and is given a special attention (like transporting a patient from one place to another, bathing of a disabled patient...), the second type is more a synchronization that prioritize a care over another which means that two or more cares cannot be done simultaneously but need to be successive in a predefined order.

As for the literature and past works dealing with synchronization, we must cite [37] where he made the aircraft routing problem a simultaneous synchronization problem that ensure that a flight possessing an Id have to depart and take off in the same time at the same place. This resulted in an approach based on a decomposition called Dantzig-Wolfe decomposition.

Synchronization constraints proposed by [36] in the operation of collecting raw milk from farms consisted in a problem called Vehicle Routing Problem with Trailer and Transshipment (VRPTT). The problem is decomposed and constituted of two main vehicles trucks that can operate alone (autonomous) and trailers that can operate only with the help of a truck (non-autonomous). The authors conceived a mathematical formulation of the VRPTT and a simple heuristic. The synchronization used in this paper is a precedence one.

Precedence constraints in the home health care problems were addressed by multiple authors such as [38] where in the context of waste collection of a household the minimization of the overall travel duration is tackled. To ensure the fluidity of the work, vehicles were separated into two types, small ones called satellites that can drive on any type of road where the second one is large or compactors that have strict and known road to drive on. A local search procedure was used in order to find feasible solution of this problem.

\section{Contribution Highlights}

In this paper, the points addressed in the literature do not handle a majority of constraints in an efficient manner, where most of the authors push the search area of solutions to grow considerably by duplicating nodes. The second point that found its limitations as the instances grows is the use of models that are based on MILP (Mixed Integer Linear Programming) where only small instances give satisfactory solutions in the contrary of large instances. Authors decided then to use metaheuristic to work on larger instances, but fail in catching the optimal solutions and are trapped in local optimum because most of them don't use local search procedure in their work in order to avoid being trapped in a local optimum. For all these points, this paper aims to contribute in:

- Put a model that engage both simultaneous and precedence constraints, caregiver lunch breaks and time windows in the same design. Afterwards solve this model with a linear solver such as Cplex.

- Manage to work with multiples and several constraints such as time windows of patients, synchronization constraints with their two type (simultaneous/precedence), caregivers skills and qualification, caregivers lunch break and the preference of patients in regard of caregivers. It is very rare to find authors that handle more than three constraints at the same time in their work.

- Propose a hybrid algorithm in order to find feasible solutions of instances considered large on existing benchmark of literatures.

\section{PROBlEM DESCRIPTION AND MATHEMATICAL FORMULATION}

The vehicle routing problem is a problem that considers the determination of routes that must be taken by vehicles from a point $\mathrm{i}$ to a point $\mathrm{j}$. The variant of the VRP considered in this study can be considered as a vehicle routing problem with time windows, synchronization, precedence and lunch break constraints (VRPTW-SPLB).

Consider $\mathrm{G}=(\mathrm{V}, \mathrm{E})$ a graph where $\mathrm{N}=\{1 \ldots \mathrm{n}\}$ is a set of clients (patients) and V= NUD a set of nodes. $c$ is the initial depot and $f$ the final one where $D=\{c, f\}$. Routes are linked by a set of points where $m=[i, j]$ a margin, $C_{i j}$ is the cost linked to the path $\mathrm{ij}$ and $\mathrm{T}_{\mathrm{ij}}$ the duration travel. A health structure offers multiple services $S=\{1 \ldots$ s $\}$ to a set of patients where a patient $i$ require a subset of services to the health structure $S_{i}=\left\{s \in S: e_{i s}=1\right\}$. A duration $d_{i s}$ is assigned to each service asked by a patient along with a time windows $\left[a_{i}, b_{i}\right]$ wherre a service cannot begin before $a_{i}$ and cannot terminate after $b_{i}$. The set of vehicles required to ensure the task of providing services to patients is denoted by $K$ where each vehicle $\mathrm{k} \in \mathrm{K}$ have a time windows of availability specified by $\alpha_{k}, \beta_{k}$. Prf $f_{i k}$ a number ensuring the preference of each vehicle $k$, this number defines the non-preferences of patient $\mathrm{i}$ in regard of the caregiver $\mathrm{k}$.

$P$ describes the lunch break of caregivers and nurses and $\mathrm{L}$ is the duration and $\left[w_{p}, z_{p}\right]$ is the time window as defined by [16]. $g_{i k}$ is 1 if the caregiver consumes his/her break at patient i before service termination and 0 otherwise, $g^{\prime}{ }_{i k}$ is 1 if the caregiver consumes his/her break at patient $\mathrm{i}$ after service termination, 0 otherwise. $s t_{i k}$ is the service start time of 
caregiver $\mathrm{k}$ at patient $\mathrm{i}$ and $\mathrm{st}_{\mathrm{pk}}$ is the lunch break time start of the caregiver $k$. For the sake of simplicity, we write $k \in K_{s}$ if $\mathrm{y}_{\mathrm{ks}}=1$ and $\mathrm{s} \in \mathrm{S}_{\mathrm{i}}$ if $\mathrm{e}_{\mathrm{is}}=1$.

For the simplification and generalization of this study, we suppose that $\mathrm{k} \in \mathrm{K}$ accomplishes a unique service $\mathrm{s}$. $\mathrm{y}_{\mathrm{ks}}$ equals 1 if vehicle $\mathrm{k}$ offers the service $\mathrm{s}, 0$ otherwise $(\forall \mathrm{k} \in \mathrm{K}, \forall \mathrm{s} \in$ S).

For customers (patients) whom are demanding more than one service either in a specific order or simultaneously, we define for each patient $\mathrm{i} \in \mathrm{N}$ a slot isr $_{\text {as }}$ the time separating the time start of service $s$ and service $r$ demanded by patient $i$ where services $\mathrm{s}$ and $\mathrm{r}$ have to be provided in a given order (service s before service $\mathrm{r}$ ).

Just to note that lunch breaks are not depending entirely on the time duration of the route, and a lunch break can be taken and utilized if the duration of the route outpaces and exceeds $\alpha_{\mathrm{p}}$. It is also permissible to take a lunch break at the end of a route even if the time window of the lunch break is not yet covered.

This problem formulation considers the minimization of the sum of non-preferences of patients towards caregivers and total travelling time by shortening to the maximum the total travelled distance overall.

To generalize the constraints of time in this formulation, we presume that all the requested services by patients are asked independently from the initial depot (center) $\mathrm{c}$ and their duration is $0\left(D_{c s}=0, \forall s \in S\right)$.

The Mixed Integer Linear Programming corresponding to our formulation is as follows:

$$
\begin{array}{r}
\min \sum_{\mathrm{i} \in \mathrm{V} \backslash\{\mathrm{f}\}} \sum_{\mathrm{j} \in \mathrm{V} \backslash\{\mathrm{c}\}} \sum_{\mathrm{k} \in \mathrm{K}} \mathrm{C}_{\mathrm{ij}} \mathrm{x}_{\mathrm{ijk}} \\
+\sum_{\mathrm{i} \in \mathrm{N}} \sum_{\mathrm{j} \in \mathrm{V} \backslash\{\mathrm{c}\}} \sum_{\mathrm{k} \in \mathrm{K}} \operatorname{Prf}_{\mathrm{ik}} \mathrm{x}_{\mathrm{ijk}}
\end{array}
$$

Subject to:

$$
\begin{aligned}
& \forall \mathrm{k} \in \mathrm{K}, \sum_{\mathrm{j} \in \mathrm{N}} \mathrm{x}_{\mathrm{cjk}}=1 \\
& \forall \mathrm{k} \in \mathrm{K}, \sum_{\mathrm{j} \in \mathrm{N}} \mathrm{x}_{\mathrm{ifk}}=1 \\
& \forall \mathrm{h} \in \mathrm{N}, \forall \mathrm{k} \in \mathrm{K}, \quad \sum_{\mathrm{i} \in \mathrm{V} \backslash\{\mathrm{f}\}} \mathrm{x}_{\mathrm{ihk}}= \\
& \sum_{\mathrm{j} \in \mathrm{V} \backslash\{\mathrm{c}\}} \mathrm{x}_{\mathrm{hjk}} \\
& \forall \mathrm{i} \in \mathrm{N}, \forall \mathrm{s} \in \mathrm{S}, \sum_{\mathrm{j} \in \mathrm{V} \backslash\{\mathrm{c}\} \quad \sum_{\mathrm{k} \in \mathrm{K}_{\mathrm{s}}} \mathrm{x}_{\mathrm{ijk}}=\mathrm{e}_{\mathrm{is}}} \\
& \forall \mathrm{i}, \mathrm{j} \in \mathrm{V}, \forall \mathrm{s} \in \mathrm{S}: \mathrm{s} \in \mathrm{S}_{\mathrm{i}} \cup \mathrm{S}_{\mathrm{j}}, \forall \mathrm{k} \in \mathrm{K}_{\mathrm{s}}, \mathrm{st}_{\mathrm{ik}}+ \\
& \left(\mathrm{T}_{\mathrm{ij}}+\mathrm{D}_{\mathrm{is}}\right) \mathrm{x}_{\mathrm{ijk}} \leq \mathrm{st} \mathrm{j}_{\mathrm{jk}}+\mathrm{b}_{\mathrm{i}}\left(1-\mathrm{x}_{\mathrm{ijk}}\right) \\
& \forall \mathrm{i} \in \mathrm{N}, \forall \mathrm{s} \in \mathrm{S}_{\mathrm{i}}, \forall \mathrm{k} \in \mathrm{K}_{\mathrm{s}}, \mathrm{a}_{\mathrm{i}} \sum_{\mathrm{j} \in \mathrm{N}} \mathrm{x}_{\mathrm{ijk}} \leq \\
& \mathrm{st} \mathrm{t}_{\mathrm{ik}} \leq \mathrm{b}_{\mathrm{i}} \sum_{\mathrm{j} \in \mathrm{N}} \mathrm{x}_{\mathrm{ijk}} \\
& \forall \mathrm{k} \in \mathrm{K}, \alpha_{\mathrm{k}} \leq \mathrm{st}_{\mathrm{ck}} \leq \beta_{\mathrm{k}} \\
& \forall \mathrm{k} \in \mathrm{K}, \alpha_{\mathrm{k}} \leq \mathrm{st}_{\mathrm{fk}} \leq \beta_{\mathrm{k}}
\end{aligned}
$$

$\forall \mathrm{i}, \mathrm{j} \in \mathrm{V}, \forall \mathrm{k} \in \mathrm{K}, \mathrm{x}_{\mathrm{ijk}} \in\{0,1\}$

$\forall \mathrm{i} \in \mathrm{V}, \forall \mathrm{k} \in \mathrm{K}, \quad \mathrm{g}_{\mathrm{ik}}, \mathrm{g}^{\prime}{ }_{\mathrm{ik}} \in\{0,1\}$

$\forall \mathrm{i} \in \mathrm{V}, \forall \mathrm{k} \in \mathrm{K}, \quad \mathrm{st}_{\mathrm{ik}} \in\{0,1\}$

The objective function (1) is to minimize the total travelling time and the sum of non-preferences. Constraints (2) and (3) ensure that each vehicle have to leave the initial depot and return to it. Constraint (4) guarantees the continuity and constancy of the routes while constraint (5) guarantees that the demands of customers (patients) are provided and executed. Scheduling and planning permitting the consistency between the durations of visits is maintained by constraint (6). Constraints (7), (8), (9) guarantee the respect of patients, caregivers time windows. Constraints (10), (11) and (12) define the nature of the decision variables.

Just to cite that the caregivers' lunch breaks are not entirely related to the time duration of the route taken, and a lunch break can be used on the condition that the duration of the route outpaces and exceeds $\alpha_{\mathrm{p}}$. It is also permitted to take a lunch break at the end of a route even if the time window of the lunch break is not yet covered.

\section{RESOLUTION APPROACH}

A metaheuristic is developed due to the challenge of solving large instances in an acceptable amount of time even though the proposed model decreases the graph size in comparison of prior formulations. The metaheuristic proposed is a hybridization of GRASP and ILS.

The GRASP method was firstly presented and dealt with by [39] for a covering set problem. The GRASP method was effective for construction routing problem, when [40] utilize it in order to solve a tour design problem with time windows to minimize the vehicle number used.

The GRASP method consists of iteration which produces in each iteration a new solution in two phases:

- Construct a feasible solution by the means of a greedy randomized heuristic.

- Improve the solution with the help a local search procedure by the iterative exploration of search space in order to improve the current solution.

The Iterated Local Search (ILS) belongs to a class of metaheuristic based on the exploration of a neighboring of near local optimum. The local optimum according to [41] is grouped in a cluster of search space. ILS initiates its search from a starting solution called initial solution of a good quality. This initial solution is created by the help of a greedy heuristic, and then improved by a local search to find a first local optimum. In the contrary of the GRASP, then a perturbation procedure is done on the current solution in each iteration in the hope to escape from the local optimum. When the solution is perturbed and improved by the local search, then this time the new solution becomes another local optimum. This cycle composed of perturbation and local search will not stop until a stopping criterion is met. 
TABLE III. PARAMETERS FOR GRASP*ILS

\begin{tabular}{|l|l|l|l|}
\hline Parameters & Notation & $\begin{array}{l}\text { Test values } \\
\text { Range }\end{array}$ & Value \\
\hline Number of restart & IterMax $_{G}$ & {$[10 . .100]$} & 45 \\
\hline Iteration number of ILS & IterMax $_{L}$ & {$[10 . .100]$} & 45 \\
\hline $\begin{array}{l}\text { Number of solution without } \\
\text { improvement }\end{array}$ & Max $_{\text {fail }}$, & {$[5 . .50]$} & 35 \\
\hline Perturbation level & pert $_{\text {max }}$ & {$[1 . .6]$} & 3 \\
\hline
\end{tabular}

\section{Results and Interpretation}

The results of experimentation of the 37 instances of each metaheuristic are found in the end of the paper, as for the summary results, they are given in Table IV and Table V. In order to have a better analysis of the obtained results, four comparisons are highlighted, one comparison for the metaheuristic GRASP*ILS with the CPLEX results, the second comparing the results of GRASP with the results of the ILS, the third comparing the GRASP with the CPLEX and finally the ILS with CPLEX. As for the representation of the results, the results are given for each group of instances $\left(\right.$ Gins $_{1}, \mathrm{Gins}_{2}, \mathrm{Gins}_{3}$ ). For CPLEX, instances that give feasible solution are denoted by $\left(\mathrm{G}_{\mathrm{cf}}\right)$ and for all instances $\left(\mathrm{G}_{\mathrm{all}}\right)$.

To understand the Table IV, the first part are the results obtained by the CPLEX solver, then the second part of the Table IV and the two parts of Table V highlight the results of the proposed methods. The performance indicators used to compare the different results are the average gap percentage of the best found solution of the GRASP*ILS method for the lower bound $\left(\operatorname{Ind}_{\mathrm{LB}}\right)$, the upper bound $\left(\operatorname{Ind}_{\mathrm{UB}}\right)$ along with each criteria separately ( $\left.\operatorname{Ind}_{\text {Moving }}\right)$ and ( $\left(\operatorname{Ind}_{\text {Pref }}\right)$. Afterwards, an exposition in seconds of the time required to obtain the best solution $\left(\mathrm{Tsec}_{\min }\right)$. The average solution of the different executions are compared with the best solution of the results obtained by the GRASP*ILS method ( $\mathrm{CObj}_{\text {Ref }}$, CMoving $\left.{ }_{\text {Ref }}\right)$ and $\mathrm{CPref}_{\mathrm{Ref}}$ ). Finally, the average computational time needed to obtain the average solutions is exposed in seconds denoted by ( $\left.\mathrm{Tsec}_{\text {average }}\right)$, as for the optimal solutions, we denote it ( Tsec $\left._{\text {optimal }}\right)$.

In the next tables, note that the results subject to the CPLEX solver which is there are no feasible solution are not included (Max time of execution is 1 hour).

Note that Ref=GRASP*ILS and $\mathrm{X}=$ Total cost of the objective function.

The CPLEX studio solver permitted to solve up to 23 optimal solution of the 37 instances tested. Meanwhile the hybrid method has solved all instances in a more reduced time (23,93 seconds for the GRASP*ILS compared to 609,53 for CPLEX).

In general, the overall results obtained show that the GRASP*ILS method is way better than CPLEX results with an average gain of $2,10 \%$, That means that this method attain an average gap of $3,19 \%$ for the lower bound in 162,24 seconds. This gap is reduced more than $1 \%$ in comparison with the CPLEX results (4,47\% of gap in 1414,51 seconds) in a computational time considerably small.

The efficiency of the hybrid method GRASP*ILS is confirmed by observing the results of the third instance group $\left(\right.$ Gins $_{3}$ ) where the hybrid method improve by $12,95 \%$ in 1186,43 seconds compared to the upper bounds of the CPLEX where the gap is nearly $23 \%$ in 3600 seconds.

Overall, the solutions presented by the hybrid method GRASP*ILS are better than the upper bound given by the CPLEX solver in 3600 seconds. In addition, the nature of the problem being NP-Hard make the CPLEX solver incapacity of producing feasible solution in a reasonable time nearly impossible for instances of more than 45 patients. In the other hand, GRASP*ILS work perfectly on larger instances.

After summarizing and analyzing the results given by the CPLEX solver and the hybrid method, an evaluation of the efficiency of the GRASP*ILS method is highlighted and compared to both the GRASP method and the ILS method.

TABLE IV. PERFORMANCE INDICATORS FOR THE 37 INSTANCES OF MILP AND GRASP*ILS

\begin{tabular}{|c|c|c|c|c|c|c|c|c|c|c|}
\hline \multirow{2}{*}{ Indicators } & \multicolumn{5}{|l|}{ MILP } & \multicolumn{5}{|c|}{ GRASP*ILS } \\
\hline & Gins $_{1}$ & $\operatorname{Gins}_{2}$ & $\operatorname{Gins}_{3}$ & $G_{c f}$ & $G_{\text {all }}$ & $\operatorname{Gins}_{1}$ & $\operatorname{Gins}_{2}$ & Gins $_{3}$ & $G_{c f}$ & $G_{\text {all }}$ \\
\hline $\operatorname{Ind}_{L B}$ & 0,00 & 2,25 & 22,90 & 4,47 & 4,47 & 0,00 & 2,01 & 11,87 & 3,19 & 3,90 \\
\hline $\operatorname{Ind}_{U B}$ & - & - & - & - & - & 0,00 & $-0,31$ & $-12,95$ & $-2,10$ & $-2,10$ \\
\hline Ind $_{\text {Moving }}$ & - & - & - & - & - & 0,00 & $-0,48$ & $-3,13$ & $-0,36$ & $-0,36$ \\
\hline Ind $_{\text {Pref }}$ & - & - & - & - & - & 0,00 & 0,15 & $-5,11$ & $-0,84$ & $-0,84$ \\
\hline$T_{s e c_{\min }}$ & 86,81 & 2002,85 & 3601,96 & 1414,51 & 1480,80 & 1,78 & 66,41 & 1186,43 & 162,24 & 344,67 \\
\hline$C X_{\text {Ref }}$ & - & - & - & - & - & 0,03 & 3,03 & 2,73 & 1,93 & 343,12 \\
\hline CMoving $_{\text {Ref }}$ & - & - & - & - & - & 0,04 & $-0,38$ & 0,49 & 0,04 & 0,04 \\
\hline CPref $_{\text {Ref }}$ & - & - & - & - & - & $-0,11$ & 0,57 & 0,64 & 0,32 & 0,33 \\
\hline Tsec optimal $_{\text {ot }}$ & - & - & - & - & - & 0,48 & 102,8 & 981,71 & 165,92 & 302,33 \\
\hline Tsec $c_{\text {optimal }}$ & 86,81 & 1287,59 & - & 609,53 & 609,53 & 0,39 & 57,39 & - & 23,93 & 23,93 \\
\hline
\end{tabular}


TABLE V. PERFORMANCE INDICATORS FOR THE 37 INSTANCES OF ILS AND GRASP

\begin{tabular}{|c|c|c|c|c|c|c|c|c|c|c|}
\hline \multirow{2}{*}{ Indicators } & \multicolumn{5}{|l|}{ MILP } & \multicolumn{5}{|c|}{ GRASP*ILS } \\
\hline & Gins $_{1}$ & Gins $_{2}$ & Gins $_{3}$ & $G_{c f}$ & $G_{\text {all }}$ & $\operatorname{Gins}_{1}$ & Gins $_{2}$ & Gins $_{3}$ & $G_{c f}$ & $G_{\text {all }}$ \\
\hline $\operatorname{Ind}_{\mathrm{LB}}$ & 0,39 & 6,19 & 9,83 & 4,74 & 4,98 & 0,00 & 4,94 & 17,28 & 5,53 & 6,43 \\
\hline $\operatorname{Ind}_{\mathrm{UB}}$ & 0,39 & 3,87 & $-15,33$ & $-0,65$ & $-0,65$ & 0,00 & 2,68 & $-3,35$ & 0,59 & 0,59 \\
\hline Ind $_{\text {Moving }}$ & 0,23 & $-0,08$ & $-4,69$ & $-0,67$ & $-0,67$ & 0,00 & 1,22 & $-1,53$ & 0,28 & 0,28 \\
\hline Ind $_{\text {Pref }}$ & $-0,04$ & 0,67 & $-1,18$ & 0,02 & 0,02 & 0,00 & $-0,29$ & 0,70 & $-0,03$ & $-0,03$ \\
\hline IndX $X_{\text {Ref }}$ & 0,39 & 4,13 & $-2,39$ & 1,44 & 0,95 & 0,00 & 2,93 & 6,17 & 2,51 & 2,72 \\
\hline IndMoving $_{\text {Ref }}$ & 0,23 & 0,33 & $-0,57$ & $-0,95$ & 0,00 & 0,00 & 1,68 & 2,73 & $-0,04$ & 1,35 \\
\hline IndPref $_{\text {Ref }}$ & $-0,04$ & 0,47 & $-0,06$ & 0,82 & 0,15 & 0,00 & $-0,50$ & 1,55 & 0,74 & 0,26 \\
\hline $\mathrm{Tsec}_{\min }$ & 0,18 & 64,33 & 465,32 & 106,94 & 148,98 & 0,21 & 260,49 & 1361,91 & 173,46 & 460,24 \\
\hline $\mathrm{CX}_{\mathrm{Ref}}$ & 19,78 & 14,27 & 2,17 & 14,93 & 13,08 & 0,97 & 8,73 & 8,30 & 5,76 & 5,68 \\
\hline CMoving $_{\text {Ref }}$ & 3,05 & 0,12 & 1,02 & 0,67 & 1,47 & 0,15 & 0,89 & 4,03 & $-0,07$ & 1,46 \\
\hline $\mathrm{CPref}_{\text {Ref }}$ & 1,76 & 2,38 & 7,22 & 4,39 & 3,45 & 0,45 & 1,15 & 1,62 & 1,60 & 1,01 \\
\hline Tsec $_{\text {optimal }}$ & 0,27 & 75,30 & 479,96 & 113,85 & 156,83 & 0,28 & 282,10 & 1331,94 & 181,53 & 459,76 \\
\hline
\end{tabular}

By comparing the results of the two methods GRASP and ILS with the CPLEX results, it is clear that ILS is better than GRASP where ILS show a gap of $4,74 \%$ on the lower bound in merely 106,94 seconds where the GRASP is only 5,53\% in 173,46 seconds. Thus, ILS improve the upper bound of the CPLEX by $0,65 \%$, meanwhile GRASP only obtain a gap of $0,59 \%$.

When comparing the best solutions obtained by the hybrid method GRASP*ILS (considered as references), the results show that the GRASP metaheuristic is less efficient with an approximate average gap of 2,51\%. On the other hand, ILS seems more efficient than GRASP with an approximate average gap of $1,44 \%$ from the method of reference. By seeing the ILS results, the difference is not that much with the hybrid method considering the overall execution $(0,85 \%$ of gap considering all instances).

To summarize, the results obtained lead us to say that the hybrid method is more efficient and stable than the standard version of ILS, where ILS is better than GRASP for a given number of local search calls. Finally, the last conclusion for this paper is that GRASP*ILS is better than ILS which is better than GRASP.

\section{CONCLUSION AND PERSPECTIVES}

In this paper, three metaheuristics for the VRPTW-SPLB are proposed. A GRASP method and an ILS method which use a constructive heuristic inspired by the nearest neighbor method and a local search which explore in an organized manner all the neighboring in order to find feasible solution optimally. Firstly, an investigation and overview of the problem of the vehicle routing problem with time windows, synchronization, precedence and lunch break constraints in the sector of home health care is presented. Then a mathematical formulation of the problem is proposed by the aid of the mixed integer programming language (MILP). After introducing the algorithms used to solve the problem, a numerical results section along with its analysis is foreshadowed to know which algorithm gives the better solution. By the outcome, it is clear that the GRASP and ILS method gives better results for larger instances than the
CPLEX solver, but compared to the lower bounds given by CPLEX, the GRASP method seems less efficient than ILS, this can be explained by the iterations independency realized by GRASP, for this reason the execution times are longer than ILS, but still reasonable for such a problem which is known to be NP-Hard. The combination of these two methods which result in GRASP*ILS method demonstrated that it is better in term of computational time and the quality of the results obtained. In order to complete this study as future research is to conduct statistical tests in order to establish which method is more stable and gives better results. The limitation found in this study are frequent in this problem such as ensuring the satisfaction of the patient by assigning to him a specific caregiver can lead to an increase in the overall cost which is very degrading in term of optimizing either resources and time. In order to escape from this, a multi-objective approach can aid to solve this matter. As a continuity of this study, a multi-objective approach of the same problem which is VRPTW-SPLB in home health care with genetic algorithms in order to optimize the overall movement costs and patient's satisfaction simultaneously will be conducted in a future paper.

\section{REFERENCES}

[1] Dantzig, G.B, \& Ramser, J.H. 1959a. The truck dispatching problem. Management Science, 6(1), 80-91.

[2] Toth, P., \& Vigo, D. 2014. Vehicle Routing : Problems, Methods, and Applications. Vol. 18. SIAM.

[3] Bräysi, O., Dullaert, W., \& Nakari, P. 2009. The potential of optimization in communal routing problems : case studies from finland. Journal of Transport Geography, 17(6), 484-490.

[4] Brasy, U., Gendreau, M.: vehicle Routing Problem with Time windows, part1: Route construction and local search Algorithm, Transport. Sci., 39(1)(2005), 104-110.

[5] G. A. P. Kindervater and M. W. P Savelsbergh. Vehicle routing : handling edge exchanges in E. H. L. Aarts et J. K. Lenstra (d.), Local search in combinatorial optimization. Wiley, Chichester, 311-336, 1997.

[6] Y. Nagata. Efficient evolutionary algorithm for the vehicle routing problem with time windows: edge assembly crossover for the VRPTW. IEEE Congress on Evolutionary Computation 2007: 11751182.

[7] Ait Haddadene, S.R, Labadie, N., \& Prodhon, C. 2016. A GRASP × ILS for the vehicle routing problem with time windows, synchronization and precedence constraints. Expert Syst. Appl, Vol. 66, 274-294. 
[8] Solomon, M.M. 1987. Agorithms for the vehicle routing and scheduling problems with time window constraints. Operations Research, 2(35), 254-265.

[9] Gehring, H., H*omberger, J., “A Parallel Hybrid Evolutionary Metaheuristics for Vehicle Routing Problem with Time Windows”, In Proceedings of EUROGEN99. Jyvaskyla: University of Jyvaskyla, 5764, 1999.

[10] L.D. Bodin, T. Sexton,. The multi-vehicle subscriber dial-a-ride problem. TIMS studies in M.Sc, 2, 73-86 (1986).

[11] J. Desrosiers, Y. Dumas,F. Soumis. A dynamic programming solution of the large-scale single-vehicle dial-a-ride with time windows. American Journal of Mathematical and Management Sciences, 6(3-4), 301-325 (1986).

[12] J. Desrosiers, Y. Dumas, M.M. Solomon, F. Soumis. Time constrained routing and scheduling. Handbooks in OR and M.Sc, 8, 35-139 (1995).

[13] L.M. Rousseau, M. Gendreau, G. Pesant. The synchronized vehicle dispatching problem. Citeseer (2003).

[14] L.M. Rousseau, M. Gendreau, G. Pesant. The Synchronized Dynamic Vehicle Dispatching Problem. INFOR : Information Systems and Operational Research, 51(2), 76-83 (2013).

[15] A. Coppi, P. Detti, J. Rafaelli. A planning and routing model for patient transportation in health care. Electronic Notes in Discrete Mathematics, 41, 125-132 (2013).

[16] E. Cheng, J.L. Rich. A home health care routing and scheduling problem. Technical report CAAM TR98-04, Rice University (1998).

[17] S. Bertels, T. Stefan. A hybrid setup for a hybrid scenario : combining heuristics for the home health care problem. Computers \& Operations Research. 33(10), 2866-2890 (2006).

[18] P. Eveborn, P. Flisberg,M. Rönnqvist. Laps Care - an operational system for staff planning of home care. European Journal of Operational Research, 171(3), 962-976 (2006).

[19] A. Trautsamwieser, P.Hirsch. Optimization of daily scheduling for home health care services. Journal of Applied Operational Research, 3(3), 124-136 (2011).

[20] C. Akjiratikarl, P. Yenradee, P.R. Drake. PSO-based algorithm for home care worker scheduling in the UK. Computers \& Industrial Engineering, 53(4), 559-583 (2007).

[21] K. Doerner, A. Focke, W.J. Gutjahr. Multicriteria tour planning for mobile healthcare facilities in a developing country. European Journal of Operational Research, 179(3), 1078-1096 (2007).

[22] D. Bredström, M. Rönnqvist. A branch and price algorithm for the combined vehicle routing and scheduling problem with synchronization constraints. NHH Dept. of Finance Management Science Discussion Paper (2007).

[23] D. Bredström, M. Rönnqvist. Combined vehicle routing and scheduling with temporal precedence and synchronization constraints. European Journal of Operational Research, 191(1), 19-31 (2008).

[24] R. Redjem, S. Kharraja, X. Xie, E. Marcon. Routing and scheduling of caregivers in home health care with synchronized visits. In : 9th International Conference on Modeling, Optimization \& SIMulation (2012).

[25] S. Afifi, D.C. Dang, A. Moukrim. A simulated annealing algorithm for the vehicle routing problem with time windows and synchronization constraints. Pages 259-265: Learning and Intelligent Optimization. Springer (2013).

[26] R. Redjem, E. Marcon. Operations management in the home health care services : a heuristic for the caregivers' routing problem. Flexible Services and Manufacturing Journal, 1-24 (2015).
[27] N. Labadie, C. Prins, Y. Yang. Iterated local search for a vehicle routing problem with synchronization constraints. Pages 257-263 of : ICORES 2014-Proceedings of the 3rd International Conference on Operations Research and Enterprise Systems, Angers, Loire Valley, France (2014).

[28] B. Issaoui, I. Zidi, E. Marcon, K. Ghedira. New Multi-Objective Approach for the Home Care Service Problem Based on Scheduling Algorithms and Variable Neighborhood Descent (2014).

[29] M.S Rasmussen, T. Justesen, A. Dohn and J. Larsen. 2012. The Home Care Crew Scheduling Problem: Preference-Based Visit Clustering and Temporal Dependencies. European Journal of Operational Research (3), p. 598-610.

[30] En-nahli, Laila \& Afifi, Sohaib \& Allaoui, Hamid \& Nouaouri, Issam. (2016). Local Search Analysis for a Vehicle Routing Problem with Synchronization and Time Windows Constraints in Home Health Care Services.-IFAC-Papers-OnLine.49.1210-1215. 10.1016/j.ifacol.2016.07.674.

[31] Euchi, J. 2020. Do drones have a realistic place in a pandemic fight for delivering medical supplies in healthcare systems problems? Chin.J.Aeronaut. (https://doi.org/10.1016/j.cja.2020.06.006).

[32] Liu, R., Xie, X., Augusto, V., \& Rodriguez, C. 2013. Heuristic algorithms for a vehicle routing problem with simultaneous delivery and pickup and time windows in home health care. European Journal of Operational Research, 230(3), 475-486.

[33] Ceselli, A., Righini, G., \& Tresoldi, E. 2014. Combined location and routing problems for the drug distribution. Discrete Applied Mathematics, 165, 130-145.

[34] Leandro C. Coelho, Jacques Renaud \& Gilbert Laporte (2016) Roadbased goods transportation: a survey of real-world logistics applications from 2000 to 2015, INFOR: Information Systems and Operational Research, 54:2, 79-96, DOI: 10.1080/03155986.2016.1167357.

[35] Y.Kergosien, Ch.Lente, J.C.Billaut and S.Perrin, 2013. Metaheuristic algorithms for solving two interconnected vehicle routing problems in a hospital complex, Computers \& Operations Research 40 (10), p. 25082518.

[36] Drexl, M. 2012. Synchronization in vehicle routing- A survey of VRPs with multiple synchronization constraints. Transportation Science, 46(3), 297-316.

[37] Ioachim, I., Desrosiers, J., Soumis, F., \& Bélanger, N. 1999. Fleet assignment and routing with schedule synchronization constraints. European Journal of Operational Research, 119(1), 75-90.

[38] Dep Pia, A., \& Flippi, C. 2006. A variable neighborhood descent algorithm from a real waste collection problem with mobile depots. International Transactions in Operational Research. 13. 125-141.

[39] Feo, T.A., \& Resende, M.G.C. 1995. Greedy randomized adaptive search procedures. Journal of global optimization, 6(2), 109-133.

[40] Kontoravdis, George \& Bard, Jonathan. (1995). A GRASP for the Vehicle Routing Problem with Time Windows. INFORMS Journal on Computing. 7. 10-23. 10.1287/ijoc.7.1.10.

[41] Glover, F., \& Laguna, M. 2013. Tabu Search. Springer New York.

[42] Nguyen, V. Prins, C, \& Prodhon, C. (2012). A multi-start iterated local search with tabu list and path relinking for the two-echelon locationrouting problem. Engineering Applications of Artificial Intelligence, 219, 598-610.

[43] J. Michallet, C. Prins, L. Amodeo, F. Yalaoui, G. Vitry Multi-start iterated local search for the periodic vehicle routing problem with time windows and time spread constraints on services Computers \& Operations Research, 41 (2014), pp. 196-207. 